\title{
Cambio de planes a través del tiempo para el traslado de roca en la pampa bonaerense
}

\author{
CRISTINA B AYÓN ${ }^{1}$ Y NORA FLEGENHEIMER ${ }^{2}$
}

\section{RESUMEN}

Se analizan las formas de traslado de las materias primas líticas a partir de la comparación entre núcleos de momentos tempranos y tardíos en la pampa bonaerense argentina. El objetivo es estudiar las modalidades en el transporte de roca para comprender las modificaciones en la organización de los cazadores-recolectores a través del tiempo. Esta región es especialmente adecuada para estudios de abastecimiento porque los recursos están muy localizados. La base de datos está formada por los materiales de las localidades Cerro La China y Cerro El Sombrero fechados entre 10200 y 11200 AP y una colección de superficie de la localidad El Guanaco correspondiente al Holoceno Tardío. A través del análisis de los núcleos se evidencia una notoria habilidad artesanal en ambos momentos. Pero, mientras que en los momentos tempranos el equipo era transportable, en los momentos tardíos coexistieron dos modalidades de abastecimiento de roca: por un lado se equiparon de materia prima determinados puntos del paisaje y, por otro, se proveyó de roca a individuos.

Palabras claves: líticos - núcleos - pampa argentina aprovisionamiento de materias primas.

\section{ABSTRACT}

The aim of the paper is to study rock distribution in order to understand changes in the hunter-gatherer societies through time. The study area is specially suited for the present purpose since resources are highly localized. Our data base includes artifacts from Cerro La China and Cerro El Sombrero localities dated between 10200 and 11200 BP and the surface collection of site 1 at El Guanaco locality, which corresponds to Late Holocene times. We conclude that during the early occupations transportable toolkits were in use, while during the more recent occupation two strategies of provisioning individuals and places coexisted. Also, the presence of prepared cores is evidence of a great flintknapping ability during both moments.

Key words: lithics - cores - Argentine humid pampas provisioning of raw materials.

Recibido: abril 2004. Manuscrito revisado aceptado: septiembre 2004.

1 Univer sidad Nacional Sur, 12 de Octubre y San Juan (8000), Bahía Blanca, ARGENTINA. Email: cbayon@ criba.edu.ar

2 CONICET - Municipalidad de Necochea, Area Arqueología y Antropología, C. C. 275, 7630, Necochea, ARGENTINA. Email: noraf@necocheanet.com.ar

\section{Introducción}

Los estudios sobre el abastecimiento de los recursos líticos han cobrado mucho impulso en las últimas décadas debido al predominio que tuvo el marco teórico de la organización tecnológica. Siguiendo esta tendencia, en las pampas bonaerenses de Argentina las investigaciones se centraron en la ubicación de fuentes potenciales de abastecimiento de los diferentes tipos de rocas, en la localización de canteras y talleres y en las modalidades de traslado de las rocas hacia diferentes áreas. El estado actual de la información nos permite confrontar el abastecimiento lítico entre las ocupaciones tempranas y tardías a partir de materiales procedentes de dos áreas de la provincia de Buenos Aires. Para las primeras ocupaciones utilizaremos información de las localidades $\mathrm{Ce}$ rro El Sombrero y Cerro La China, en el área serrana de Tandilia, y para el Holoceno Tardío, materiales procedentes de la localidad El Guanaco sitio 1 , un yacimiento multicomponente en el área interserrana costera. El objetivo de este trabajo es realizar un análisis de los núcleos, y a partir de ello plantear el significado de las distintas maneras en que se trasladaron las rocas. Nuestro estudio es pertinente para la discusión sobre las modificaciones en la movilidad y el acceso a los recursos ocurridas en las sociedades de cazadores recolectores regionales. Las evidencias halladas por distintos autores muestran un cambio desde grupos más móviles en el Holoceno Temprano, a grupos menos móviles, con una mayor complejidad que incluye aumento en la densidad poblacional, uso de diferentes recursos y paisajes e incremento en la territorialidad (Barrientos 1997; Martínez 1999; Politis y Madrid 2001; González de Bonaveri 2002).

\section{Caracterización de la base de recursos lítica regional}

La pampa bonaerense es una extensa llanura con pocos afloramientos rocosos. La base de recursos lítica regional presenta tres características de re- 
levancia para los estudios de abastecimiento. La primera de estas características es que los afloramientos se encuentran muy localizados. Los más importantes están constituidos por dos sistemas serranos: el sistema de Tandilia al norte, y el de Ventania, al sur, con escasos afloramientos menores entre ambos (Figura 1). También es posible obtener rocas en la costa atlántica, pero éstas se encuentran dispersas, ya que fueron transportadas por las corrientes marinas y reconcentradas en las playas.
La segunda, es la posibilidad de diferenciar las rocas de cada una de estas áreas de abastecimiento. Esto es particularmente importante en el caso de las rocas cuarcíticas porque proceden de ambos sistemas serranos, y son las de mayor interés arqueológico. En trabajos previos planteamos que estas rocas se pueden distinguir petrográficamente ya que las del sistema de Tandilia son sedimentarias (ortocuarcitas) y las del sistema de Ventania son metamórficas (metacuarcitas) (Bayón et al. 1999). Las materias primas disponibles en la cos-

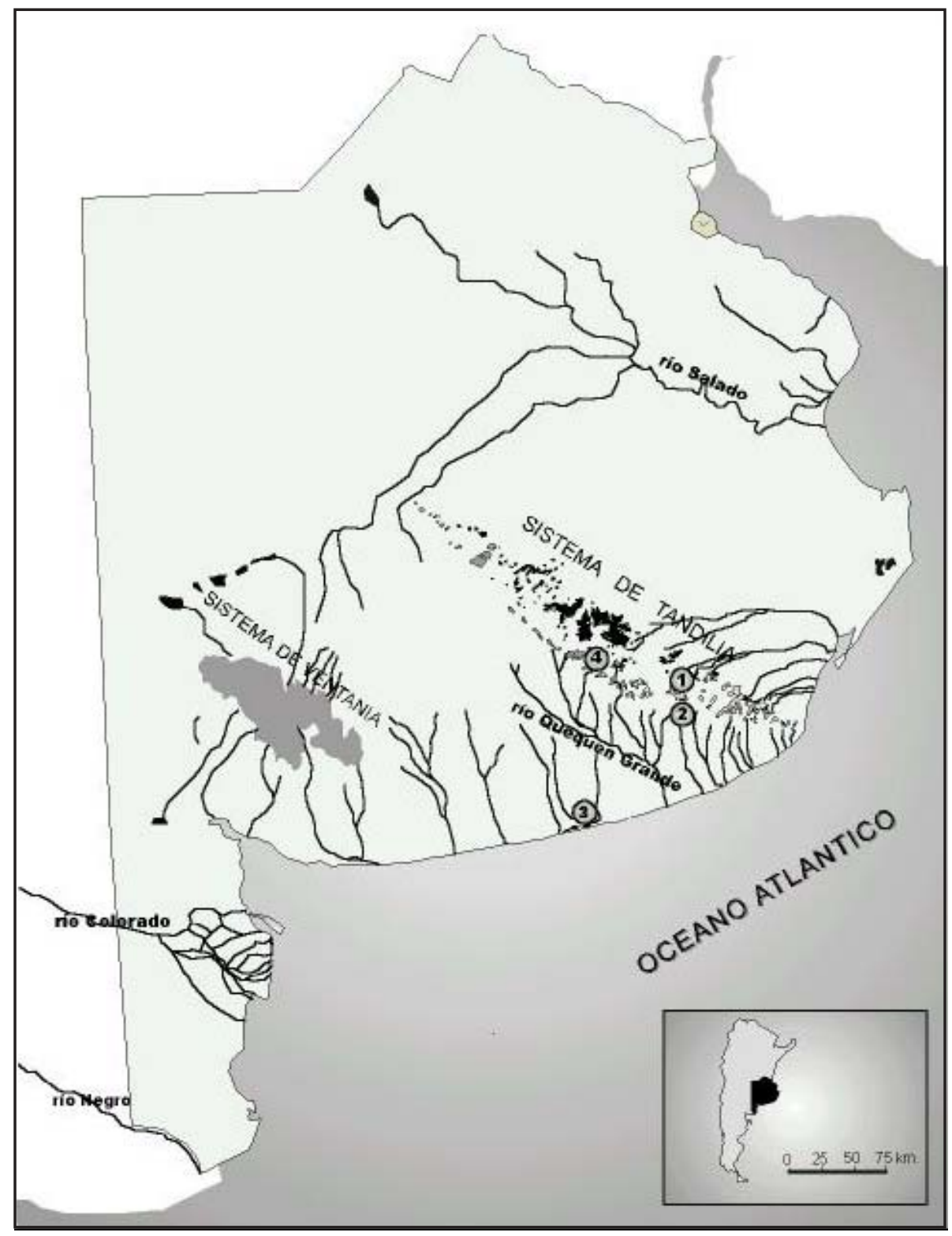

Figura 1. Mapa de ubicación de los sitios y fuentes de materia prima: 1) Cerro El Sombrero; 2) Cerro La China; 3) El Guanaco; 4) Arroyo Diamante. 
ta son rodados de basalto y sílices muy característicos.

La tercera, es que la calidad para la talla de las diferentes variedades de rocas dentro de cada sistema serrano y entre éstos, es muy distinta. Aunque todas las variedades de rocas tallables han sido usadas, su diversidad influyó en las decisiones de abastecimiento y en el esfuerzo realizado para su traslado.

En el sistema de Tandilia se reconocen tres formaciones. El complejo Buenos Aires (ver Figura 1) está compuesto principalmente de rocas ígneas y ha aportado cuarzos para la talla. El Grupo Sierras Bayas proporcionó ortocuarcitas de muy buena calidad. También componen este grupo ftanitas y dolomías silicificadas, de buena calidad. En el sistema serrano los afloramientos del Grupo Sierras Bayas (GSB) están muy localizados. Además, dentro de éstos, las ortocuarcitas presentan una silicificación variable, lo que hace que su calidad sea irregular y las porciones más aptas para la talla aparecen en forma muy circunscrita aún y hasta ahora sólo se han ubicado en el sector oriental de los afloramientos del GSB (ver Figura 1). El área de canteras más densa conocida en la región está en el valle del Arroyo Diamante, y se vincula con un intenso aprovechamiento del recurso. En este sitio se realizó un fechado que nos permitió proponer que la cantera fue utilizada desde hace al menos 5000 años (Flegenheimer $e t$ al. 1999), aunque el mayor volumen de roca fue explotado durante el Holoceno Tardío. En tanto, la Formación Balcarce está compuesta de ortocuarcitas de calidad media.

Las rocas de Ventania tienen fuertes evidencias de deformación debido a procesos metamórficos intensos, y estos rasgos han afectado su calidad para la talla, haciendo que sea menor que las ortocuarcitas del GSB de Tandilia. Los rodados costeros, por su parte, suelen ser de buena calidad, pero su forma y tamaño condicionan las maneras de reducción utilizables. Por esa razón, fueron casi exclusivamente aprovechados por talla bipolar (Flegenheimer et al. 1995).

Diversos autores han identificado fuentes potenciales y canteras de estas rocas de interés arqueológico (p.e., Politis 1984; Oliva y Barrientos 1988; Flegenheimer 1991; Lozano 1991; Madrid y Salemme 1991; Mazzanti 1993; Franco 1994;
Pupio 1996; Flegenheimer et al. 1996; Bayón y Zavala 1997; Oliva y Moirano 1997; Bayón et al. 1999; Ormazábal 1999; Madrid et al. 2000; Moirano 2000; Bonomo 2002; Flegenheimer y Bayón 2002; Messineo 2002; Messineo et al. 2002; Barros y Messineo 2004).

En resumen, desde el punto de vista del esfuerzo del traslado, las diferentes variedades de rocas tuvieron distinta importancia. Hay una roca cuyo abastecimiento fue de importancia regional: la ortocuarcita del GSB. Esta roca es mayoritaria en muchos contextos en el norte y centro de la provincia de Buenos Aires. Por ejemplo, la encontramos como la roca más representada en los sitios serranos de Tandilia, distantes $60 \mathrm{~km}$ hacia el este de las fuentes (Flegenheimer y Bayón 1999; Valverde 2002); también en sitios en la depresión del río Salado a $220 \mathrm{~km}$ al norte de esta cantera (Bonaveri et al. 1999); o en la localidad El Guanaco, distante poco más de $100 \mathrm{~km}$ al sur. Como roca secundaria está presente en casi todos los contextos sobre la costa y en el oeste de la provincia. También aparece, siempre en pequeñas cantidades, al sur de Ventania, distante unos $275 \mathrm{~km}$, y hasta en sitios del río Colorado y el Negro a más de $400 \mathrm{~km}$. Finalmente, hay rocas como las riolitas, las metacuarcitas de Ventania, los rodados costeros y las ftanitas en el extremo occidental de Tandilia que fueron la principal materia prima de importancia local en un radio de hasta $60 \mathrm{~km}$ de la fuente (de importancia local), y fueron trasladadas por largas distancias con menor frecuencia.

Para la discusión de los planes de abastecimiento, en este trabajo usamos los criterios tomados de Meltzer (1989: 33) y modificados para que sean útiles a la escala espacial de la pampa bonaerense. Cuando hablamos de rocas inmediatamente disponibles nos referimos a aquellas que están accesibles en un radio de alrededor de $10 \mathrm{~km}$ del sitio. Consideramos que las rocas que se obtienen en un radio de hasta $60 \mathrm{~km}$ son locales, las que se encuentran entre 60 y $100 \mathrm{~km}$ son de distancia media, y las que provienen de más de $100 \mathrm{~km}$ son de largas distancias.

\section{La base de datos}

A partir de la década de los 80 las investigaciones en la región pampeana se han incrementado constantemente. Distintos equipos trabajan en las 
diferentes subregiones y áreas. Esta actividad produjo una información regional muy importante. En la actualidad se han fechado unos 50 sitios en la región que presentan ocupaciones desde fines del Pleistoceno hasta el Holoceno Tardío. En una síntesis reciente se plantean las principales características de las ocupaciones y de los cambios conocidos en este lapso (Politis y Madrid 2001). Nuestra base de datos en este trabajo está conformada por los conjuntos líticos producidos por distintas sociedades de cazadores recolectores de la pampa bonaerense. Como dijimos, haremos referencia a sitios ubicados dentro de dos áreas de esta vasta región.

\section{Localidades serranas}

Las localidades Cerro La China y Cerro El Sombrero se encuentran en el ambiente serrano de Tandilia y en ellas se han excavado tres y dos sitios, respectivamente. Las ocupaciones más antiguas están fechadas en la transición PleistocenoHoloceno (Flegenheimer y Zárate 1997). Existe una importante variabilidad intersitio y se ha propuesto distinta funcionalidad para cada uno de ellos a partir de los conjuntos líticos y su emplazamiento en el paisaje. El sitio más denso es la Cima de Cerro El Sombrero, considerado como sitio de reequipamiento y avistadero. El sitio menos denso es el Sitio 2 de Cerro La China donde se infieren actividades de caza. Los tres restantes son considerados sitios de actividades domésticas y de ellos provienen casi todos los núcleos estudiados.

Los contextos están formados por una variedad muy grande de artefactos formatizados que incluyen, entre los más representativos: puntas "cola de pescado", raederas, cuchillos, denticulados y cepillos (Flegenheimer et al. 2003). La mayoría fueron confeccionados por retoque unifacial, aunque la talla bifacial llega a tener una presencia importante, sobre todo en la Cima de Cerro El Sombrero (40\%), incluso algunos artefactos están formatizados sobre lascas de reducción bifacial. También la talla bipolar está claramente representada en el conjunto, y existe un bajo porcentaje de piezas elaboradas por picado, abrasión y pulido, como, por ejemplo, una pieza discoidal decorada, semejante a las halladas en otros contextos sudamericanos tempranos (Bird 1970).

Para la confección de los artefactos tallados se usaron materias primas con trayectorias diferentes. Por un lado, se usaron, dentro de una estrategia expeditiva, algunas rocas inmediatamente disponibles como los cuarzos del basamento y las ortocuarcitas de la Formación Balcarce (Flegenheimer et al. 2004). Por otro, la mayor parte del instrumental $(87 \%)$ está manufacturado sobre ortocuarcitas del GSB (Flegenheimer y Bayón 1999), que, como ya dijimos, es una roca de abastecimiento local, encontrándose las fuentes en un radio de $60 \mathrm{~km}$ de los sitios. La manera de utilizar esta roca local indica comportamientos de cuidado y economización de la materia prima a través del mantenimiento y reciclaje de instrumentos (Odell 1996). Hay además un aspecto interesante en la provisión de las ortocuarcitas GSB y es que fueron seleccionadas por color. En las canteras de la región esta roca es predominantemente blanquecina, pero en los contextos tempranos el $60 \%$ es coloreada (Flegenheimer y Bayón 1999). La tercera trayectoria está representada por escasos artefactos tallados sobre una roca transportada por larga distancia: se trata de una caliza silicificada que proviene de Uruguay (Flegenheimer et al. 2003).

En síntesis, los grupos tempranos basaron su abastecimiento en la roca local de mejor calidad y realizaron un esfuerzo adicional por seleccionar la coloreada, esto lo hemos interpretado como de valor simbólico o social (Flegenheimer y Bayón 1999). En segundo lugar, usaron rocas de disponibilidad inmediata, de calidad inferior, y cuya forma de manejo difiere de la anterior: fue usada de manera poco intensiva, los artefactos fueron confeccionados informalmente, tienen mayores dimensiones y más corteza. Finalmente, hay escasos instrumentos tallados sobre rocas que fueron trasladadas por centenares de kilómetros y que interpretamos como bienes que viajaron dentro de redes sociales amplias.

\section{Localidad en la llanura}

El sitio 1 de la localidad El Guanaco se encuentra en plena llanura pampeana, entre ambos sistemas serranos, y a $13 \mathrm{~km}$ del mar (Bayón et al. 2004). Se trata de un sitio multicomponente emplazado en una loma suave en las inmediaciones de la laguna El Lucero. El sitio fue ocupado desde el Holoceno Temprano hasta el Holoceno Tardío (Bayón et al. 2004).

En este trabajo discutiremos los materiales líticos tallados procedentes del horizonte Ap del suelo, 


\begin{tabular}{|c|c|c|c|c|c|}
\hline & $\begin{array}{l}\text { Sistema de Tandilia } \\
\text { Sierras Bayas }\end{array}$ & Ftanita & Costa & $\begin{array}{l}\text { Sistema de } \\
\text { Ventania }\end{array}$ & Varios \\
\hline Unifacial & 185 & 12 & 11 & 14 & 14 \\
\hline Bifacial & 12 & 0 & 1 & 3 & 1 \\
\hline Desechos & 243 & 11 & 43 & 8 & 15 \\
\hline Núcleos & 21 & 3 & Bipolares 75 & 1 & 0 \\
\hline
\end{tabular}

Tabla 1. Representación de materias primas entre los artefactos tallados del sitio El Guanaco (Colección Márquez).

es decir, un horizonte A de suelo modificado por las tareas agrícolas, removido por el arado. Cronológicamente, basándonos en los trabajos geoarqueológicos, en los fechados radiocarbónicos y en el contexto arqueológico, estos materiales se adscriben al Holoceno Tardío, anterior al contacto hispano-indígena. Los cinco fechados realizados sobre restos humanos hallados inmediatamente por debajo del horizonte A oscilan entre 1738 y 2470 años AP (Flegenheimer et al. 2002; Bayón et al. 2004; Mazzia et al. 2004). Esto daría el límite temporal inferior, en tanto el superior se basa en que las evidencias arqueológicas de ocupaciones posteriores al contacto hispano-indígena son muy escasas, habiéndose recuperado únicamente una cuenta de vidrio de origen europeo y pocos fragmentos de botellas de bebidas alcohólicas.

Como la localidad está lejos de los afloramientos, toda la roca necesariamente fue trasladada. La colección reúne materiales manufacturados sobre rocas de Tandil, Ventania y la costa (Tabla 1). La variedad de instrumentos es grande e incluye yunques, percutores, bolas de boleadora, una punta mediana, raederas, raspadores, cuchillos, perforadores, molinos y manos. Hay un amplio predominio de la talla unifacial y una alta frecuencia de bipolaridad, aunque también se registraron bifaces y algunas hojas. Casi la mitad de los artefactos unifaciales están sumariamente retocados y pueden ser considerados informales. Lo más destacable de esta colección de superficie es el volumen de la roca transportada, ya que hemos registrado $146 \mathrm{k}$ de roca, en artefactos de trayectorias muy variadas. Una gran proporción de este peso lo conforman los instrumentos formalizados por picado, abrasión y pulido, especialmente los de molienda, y que no trataremos en este trabajo. La roca más empleada para los artefactos manufacturados por talla fue la ortocuarcita GSB (34 k) que debió trasladarse por más de $100 \mathrm{~km}$ desde Tandil. En segundo lugar, se usaron los rodados costeros transportados por $13 \mathrm{~km}(12.5 \mathrm{k})$. Son las rocas más cercanas al sitio o locales y fueron aprovechados mediante talla bipolar.

En síntesis, el suministro de rocas muestra un área de abastecimiento variada que incluye ambos sistemas serranos y la costa y que se articula claramente con una estrategia de acopio de materias primas y artefactos. A partir de la concentración y el volumen de materiales hemos propuesto que la localidad constituyó un ámbito calificado del paisaje ocupado reiteradamente (Bayón y Fleg enheimer 1998).

Los núcleos

En este trabajo consideramos como núcleo al artefacto lítico en el que podemos distinguir al menos una boca de lascado clara que da inicio a una lasca útil como forma base. Es decir, quedan excluidos los fragmentos de núcleos que no conserven el inicio de algún lascado. Tampoco tomaremos en cuenta en este trabajo a los núcleos bipolares, ya que tienen una trayectoria de producción muy particular. Sólo queremos aclarar que ambos conjuntos, temprano y tardío, incluyen núcleos bipolares.

Para abordar el estudio de los núcleos registramos las siguientes variables propuestas por Aschero (1975 Ms): forma total de la pieza, entero/fragmento, largo, ancho y espesor, tipo de roca, forma base, número de planos de percusión, forma de los planos de percusión y ángulo complementario de percusión. También hemos registrado el peso del núcleo, la cantidad de negativos de lascados en los que se observa la boca de extracción, el tamaño (largo y ancho) de estos negativos, la presencia de negativos anteriores sin boca de extracción visible, el porcentaje de corteza en relación a la superficie total, el tipo de corteza y las señales de apoyo, terminaciones fallidas ${ }^{3}$ y

\footnotetext{
3 En inglés, "hinge".
} 
golpes fallidos. Hemos dividido el conjunto de núcleos en tres tamaños según su peso: pequeños hasta $100 \mathrm{~g}$; medianos entre 101-500 g; y grandes, los mayores de $500 \mathrm{~g}$.

En las excavaciones de los sitios serranos hemos registrado un total de 27 núcleos tallados por percusión directa, la mitad de ellos provienen de un sitio de actividades domésticas (Cerro La China Sitio 3).

Separamos los núcleos en dos grupos de acuerdo a la variedad de roca y a la distancia a la fuente de abastecimiento: a) locales, en este caso tallados en ortocuarcita GSB ( $n=8)$; y b) sobre rocas inmediatamente disponibles: ortocuarcita Balcarce $(n=15)$ y cuarzo $(n=4)$.

Una de las primeras apreciaciones es que existe una diferencia de tamaño entre los dos subconjuntos de núcleos (Figura 2 a y b). Mientras que los núcleos pequeños están confeccionados en rocas locales y en las inmediatamente disponibles, los medianos sólo están registrados en estas últimas. Esta situación se ve claramente reflejada en el peso de núcleos (ver Tabla 2), ya que todos los núcleos sobre ortocuarcita GSB, salvo un ejemplar fragmentado, pesan menos de 50 g. En cambio, los tallados sobre rocas inmediatamente disponibles varían entre 40 y $500 \mathrm{~g}$.

En cuanto a la presencia de corteza, hemos registrado que los núcleos de rocas inmediatamente disponibles presentan reserva de corteza, y salvo uno está totalmente limpio. Mientras que los locales rara vez conservan su corteza, salvo un caso con toda una cara con corteza.
La mayoría de los núcleos sobre las rocas inmediatamente disponibles son carecen de formas tipificables $(n=13)$, sólo seis presentan formas más regulares, clasificables como: cuatro piramidales irregulares (Figura 2a), uno discoidal irregular y uno poliédrico. En cambio, entre los núcleos sobre rocas locales predominan los de formas más regulares, hay dos de formas no tipificables, uno poliédrico, dos discoidales irregulares, uno discoidal regular (Figura 2b), uno piramidal irregular, e incluso un prismático irregular. $\mathrm{Al}$ menos tres muestran señales de talla con apoyo (Figura $2 b)$. Algunos de estos núcleos en ambos subconjuntos presentan plataformas preparadas para la extracción de lascas y un frente de extracción regularizado. Pero esta preparación es más frecuente y más intensa en los núcleos sobre la ortocuarcita GSB, lo cual es de esperar ya que se encuentra más distante de los sitios y es de mejor calidad para la talla.

Hemos comparado el registro del tamaño de los negativos de lascado de los núcleos con el tamaño de los instrumentos formalizados recuperados en los sitios. Hallamos que las lascas que se extrajeron de estos núcleos son más pequeñas que la mayor parte de las formas base de los instrumentos. Es decir, que aunque los núcleos fueron formatizados para extraer lascas pequeñas, éstas no se emplearon como formas base de la mayoría de los instrumentos retocados.

En la colección de superficie del Holoceno Tardío se han hallado 25 núcleos. El conjunto incluye las siguientes materias primas: 21 núcleos de ortocuarcitas GSB (84\%), tres de ftanita y uno de metacuarcita de Ventania. Están representados los

\begin{tabular}{|c|c|c|c|}
\hline \multicolumn{2}{|c|}{ Materia prima } & Local & Inmediatamente disponible \\
\hline \multicolumn{2}{|l|}{ Cantidad } & 8 & 19 \\
\hline \multicolumn{2}{|c|}{ Tamaño y peso } & Pequeños, hasta 50 & Pequeños y medianos hasta 500 \\
\hline \multicolumn{2}{|c|}{ Corteza } & Poca o nada & Poca o mucha \\
\hline \multicolumn{2}{|c|}{ Plataformas de percusión } & Lisas & Lisas y naturales \\
\hline \multicolumn{2}{|c|}{ Formas } & Más regulares & $68 \%$ amorfos \\
\hline \multirow[t]{2}{*}{$<50$ grs } & Preparados & 4 & 0 \\
\hline & No preparados & 3 & 1 \\
\hline \multirow[t]{2}{*}{$50-500$ grs } & Preparados & 0 & 2 \\
\hline & No preparados & 1 & 16 \\
\hline
\end{tabular}

Tabla 2. Cuadro comparativo de núcleos tempranos (Cerro La China y Cerro El Sombrero). 


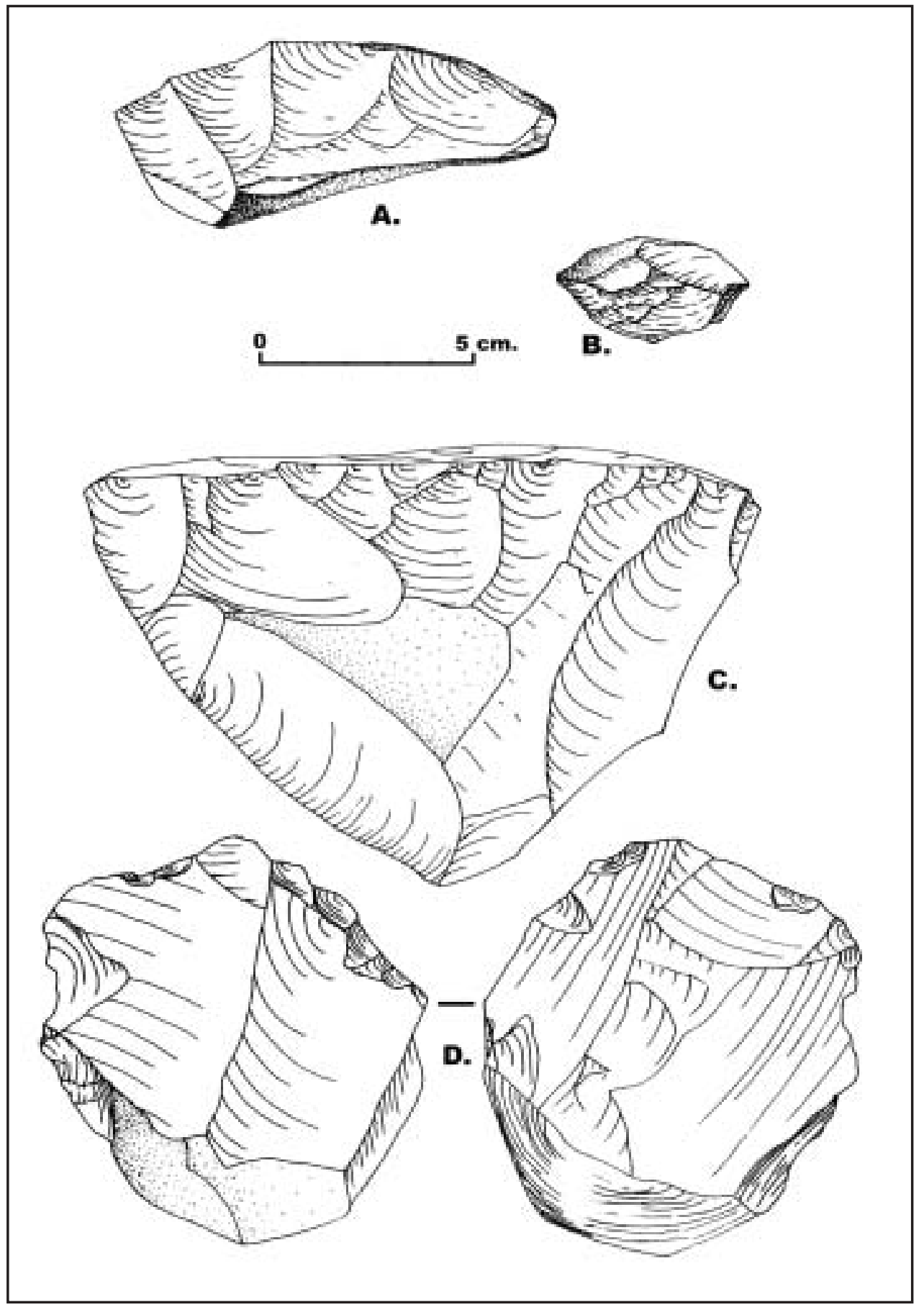

Figura 2. a) Núcleo pseudopiramidal de ortocuarcita Formación Balcarce, Cerro La China Sitio 3 nº 1556; b) núcleo discoidal regular de ortocuarcita G.S.B., Cerro La China Sitio $1 \mathrm{n}^{\circ}$ 2880; c) núcleo piramidal de ortocuarcita G.S.B., Col. Márquez, El Guanaco nº 1300; d) núcleo discoidal de ortocuarcita G.S.B., Col. Márquez, El Guanaco nº 310.

tres tamaños: pequeños $(6 / 25)$, medianos $(8 / 25)$ y grandes (11/25), tanto entre los pequeños como entre los grandes predominan las ortocuarcitas
GSB. La presencia de corteza es baja. El 48\% de los núcleos de ortocuarcita GSB no presentan corteza y sólo dos ejemplares tienen $30 \%$ y $50 \%$ de 
corteza. Este hecho tiene su correlato entre las lascas ya que sólo el $6 \%$ conserva algo de corteza en su cara dorsal (14/25). Esto permite inferir que la materia prima era trasladada preferentemente limpia. Los tres núcleos de ftanita tienen entre un $20 \%$ y un $60 \%$ de corteza. En cuanto a las formas, los núcleos piramidales son los más frecuentes $(36 \%)$ (Figura 2c), en segundo lugar los poliédricos $(28 \%)$, seguidos por los discoidales (20\%) (Figura 2d), amorfos (12\%), los de forma no tipificable (12\%) y los bifaciales (4\%). Seis núcleos presentan claras evidencias de haber sido apoyados para realizar las extracciones. Los núcleos con preparación de plataformas, del frente de extracción y de las aristas que guían las extracciones posteriores, constituyen el $64 \%$ del conjunto y están presentes en los tres tamaños (Tabla 3). Este acondicionamiento es más fuerte en los confeccionados sobre ortocuarcita del GSB (14/21). Aunque la muestra de núcleos de ftanita y metacuarcita es pequeña, se observa una tendencia a presentar plataformas menos preparadas y una morfología más irregular. Los núcleos pequeños fueron todos preparados. En cambio, los núcleos medianos presentan la menor formatización de plataformas y frentes.

En el contexto lítico han sido recuperadas unas pocas hojas, que también están presentes en varios sitios pampeanos (Bayón et al. 1999; Nami 1999). No hemos registrados verdaderos núcleos prismáticos en el sitio, pero consideramos que algunos núcleos atípicos podrían estar preparados para la extracción de escasas hojas.
En síntesis, los núcleos grandes y pequeños fueron los más cuidadosamente manufacturados. Los grandes presentan ángulos y frentes de extracción limpios y activos, sería posible continuar extrayendo lascas, es decir, fueron descartados con una vida útil larga. Sólo en el subgrupo de los núcleos pequeños, algunos están agotados con ángulos rectos y obtusos y terminaciones fallidas.

\section{Discusión}

Hemos hallado coincidencias significativas en el tratamiento de los núcleos de ambos conjuntos. En los dos hay una elección preferencial de la roca de mejor calidad y un tratamiento diferente según la calidad de la materia prima (Tablas 2 y $3)$. Hay una mayor inversión de trabajo en los núcleos confeccionados con ortocuarcita GSB que era la mejor materia prima disponible en la pampa bonaerense. Los núcleos en ortocuarcita GSB generalmente presentan plataformas y frentes de extracción preparados cuidadosamente, en tanto que los realizados en rocas de calidad inferior fueron menos elaborados. Basándonos en esta preparación de los núcleos proponemos que hay una habilidad artesanal sostenida en el tiempo, que para el caso de las ocupaciones más tempranas, está además claramente representada por la talla de puntas "cola de pescado".

A la vez, hemos encontrado importantes diferencias en las modalidades de abastecimiento del recurso lítico. En los sitios tempranos los núcleos están presentes por descarte, aunque algunos

\begin{tabular}{|c|c|c|c|c|}
\hline \multirow{2}{*}{\multicolumn{2}{|c|}{ Materia prima }} & \multicolumn{3}{|c|}{ Aprovisionamiento en un radio mayor a $150 \mathrm{~km}$} \\
\hline & & Ortocuarcita SB & Ftanita & Metacuarcita \\
\hline \multicolumn{2}{|l|}{ Cantidad } & 21 & 3 & 1 \\
\hline \multicolumn{2}{|c|}{ Tamaño y peso } & Pequeños, medianos y grandes & Medianos & Mediano \\
\hline \multicolumn{2}{|c|}{ Corteza } & Poca o nada & Moderada & Nada \\
\hline \multicolumn{2}{|c|}{ Plataformas de percusión } & Lisas y lisas preparadas & Naturales y lisas & Naturales \\
\hline \multicolumn{2}{|c|}{ Formas } & Más regulares & Amorfos y poliédricos & Bifacial \\
\hline \multirow[t]{2}{*}{$<100 \mathrm{grs}$} & Preparados & 6 & 0 & 0 \\
\hline & No preparados & 0 & 0 & 0 \\
\hline \multirow[t]{2}{*}{$100-500$ grs } & Preparados & 3 & 0 & 1 \\
\hline & No preparados & 1 & 2 & \\
\hline \multirow[t]{2}{*}{$>500 \mathrm{grs}$} & Preparados & 6 & 0 & 0 \\
\hline & No preparados & 5 & 1 & 0 \\
\hline
\end{tabular}

Tabla 3. Cuadro comparativo de núcleos tardíos (sitio El Guanaco, Colección Márquez). 
muestran fuentes sin terminaciones fallidas que por sus ángulos podrían ser activos, su tamaño reducido debió ser el motivo del abandono. La mayoría son demasiado pequeños para producir formas base como las que se han usado para la confección de instrumentos. El hecho de que los tamaños de las formas base de los instrumentos sean mayores que los de los últimos negativos de lascado, nos permite proponer que los núcleos se usaban hasta quedar agotados, y que además de núcleos, se pueden haber trasladado artefactos ya tallados o lascas. En trabajos anteriores, a partir de la existencia de lascas de reducción bifacial medianas/grandes usadas como formas base de instrumentos, propusimos que también se estaban transportando grandes bifaces (Flegenheimer 2001).

En la colección del Holoceno Tardío encontramos que tanto los núcleos pequeños como los muy grandes están cuidadosamente preparados, en tanto que los medianos lo han sido en menor proporción. Si dejamos de lado la posibilidad de que esta situación pueda deberse a un problema de muestra, la podemos explicar por al menos dos procedimientos: a) que los núcleos formalizados pequeños provengan del uso intensivo de los grandes $o, b)$ que se haya buscado producir núcleos de diferente tamaño para obtener lascas distintas. Entonces, nos preguntamos qué esperaríamos encontrar si se tratara del primer caso y estuviéramos ante una secuencia de reducción de los núcleos muy grandes a los pequeños o, por el contrario, qué esperaríamos si fueran el resultado de dos diseños distintos. En el primer caso, si los núcleos pequeños fueran los grandes agotados, los medianos deberían corresponder a una etapa intermedia de reducción y, como ya señalamos, no es así. Tampoco se han hallado lascas de reactivación de las plataformas que serían esperables si se hubiera intentado mantener una forma regular de los núcleos durante toda la reducción. Por ello, consideramos que los datos disponibles no permiten sustentar esta primera proposición.

El segundo procedimiento daría como resultado un conjunto de núcleos grandes y otro de núcleos pequeños; cada conjunto con su particular historia de vida útil, y esto parece concordar más con los datos disponibles, ya que encontramos núcleos grandes descartados en perfecto estado, sin terminaciones fallidas, ni embotamientos de ángulos, aptos para realizar muchas extracciones y núcleos pequeños agotados y no agotados. Respecto de los medianos, el único ejemplar piramidal disponible podría ser la forma inicial de los pequeños; la falta de otros ejemplares impide avanzar más allá.

En suma, consideramos que los tamaños de estos núcleos tardíos posiblemente no corresponden a distintos momentos de la vida útil del mismo artefacto, sino que se manufacturaron núcleos de distintas dimensiones para obtener lascas de diferentes tamaños.

Pensamos que los núcleos grandes representan casos de almacenaje de materia prima en sitios predeterminados, por lo que consideramos que la planificación evidenciada es la de aprovisionamiento del lugar (Kuhn 1995), en este caso a través del traslado de núcleos y de grandes lascas nodulares. Martínez (1999) ha destacado esta forma de suministro de materia prima en sitios tardíos a lo largo del río Quequén Grande en el área interserrana y ha propuesto que constituyó una estrategia de litificación del paisaje que distribuye socialmente el recurso en lugares donde naturalmente no estaba disponible (Martínez y Mackie 2003/2004). Esta modalidad es la misma que puede observarse en colecciones privadas de materiales de superficie en otros sitios del área interserrana.

Pero no es la única planificación de abastecimiento que se utilizó en el Holoceno Tardío. Bayón y colaboradores (2000 Ms) han destacado que en ese lapso los grupos pampeanos implementaron diferentes planes para aprovisionarse de rocas. De este modo, coexistieron la modalidad de almacenaje aquí descrita como una forma de cuidado y el aprovechamiento intensivo del recurso, éste es el caso del área del Salado (González de Bonaveri et al. 1999).

Los núcleos pequeños que fueron descartados agotados posiblemente representen otro aspecto organizativo que tiene que ver con el equipamiento de los individuos. ${ }^{4}$ Como en el caso de los núcleos tempranos, este equipamiento personal fue confeccionado para extraer lascas medianas y pequeñas, que sería la materia prima que la gente trasladaba con ella.

\footnotetext{
4 "Personal gear" sensu Binford (1979) у "provisioning individuals", sensu Kuhn (1995).
} 


\section{Conclusiones}

Durante las ocupaciones tempranas la materia prima de mejor calidad se acarreó por decenas de kilómetros en forma de instrumentos, lascas o tal vez como núcleos medianos que sólo se descartaron cuando la masa disponible se agotaba, y en menor medida también como bifaces. Estas formas de traslado de roca para el poblamiento temprano avalan la visión actual de grupos altamente móviles con un equipo instrumental muy transportable (p.e., Borrero y Franco 1997; Politis y Madrid 2001; Miotti y Cattáneo 2003).

Para los momentos del Holoceno Tardío en la pampa bonaerense, varios autores, a partir de distintos indicadores, han sugerido un decrecimiento de la movilidad y un incremento de la población relacionados a una mayor complejidad social (Barrientos 1997; González de Bonaveri et al. 1999; Politis y Madrid 2001; Martínez y Mackie 2003/2004, entre otros). Tecnológicamente, es frecuente vincular este proceso con una mayor expeditividad en los contextos líticos, y sobre todo en los núcleos (p.e., Parry y Kelly 1987; Jeske 1987; Koldehoff 1992). El componente tardío del sitio El Guanaco muestra una situación peculiar, que coincide parcialmente con esta propuesta: por un lado, la formatización de instrumentos es escasa y básicamente unifacial, pero en cambio, se realiza un enorme esfuerzo tecnológico en el abastecimiento, trasladando núcleos con una gran inversión de trabajo en su preparación. Para interpretar esta estrategia, la clave reside en la preparación de los grandes núcleos formales, que están claramente formatizados para no fracasar en ninguna extracción. No sólo se abastece un lugar porque el reg reso es proyectado y previsible, sino que se invierte mucho trabajo en la confección de los núcleos. Creemos que este esfuerzo inicial indica que el acceso a las canteras pudo haber sido limitado. En el caso pampeano, donde no hay barreras geográficas ni impedimentos climáticos que limiten la obtención de las materias primas, es razonable pensar que la dificultad para el abastecimiento estuvo en un control social de las canteras. Probablemente existió una necesidad de negociación por el recurso como consecuencia de la extremada localización de la materia prima de buena calidad, que impondría este esfuerzo mayor para el aprovisionamiento de roca (Flegenheimer y Bayón 2002). A partir de lo expuesto, queda claro que el aprovisionamiento lo debieron hacer los artesanos más hábiles, que podían garantizar a través de los núcleos el máximo aprovechamiento del volumen de roca transportada.

\section{REFERENCIAS CITADAS}

ASCHERO, C., 1975 Ms. Ensayo para una clasificación morfológica de artefactos líticos aplicada a estudios tipológicos comparativos. Informe de Investigación presentado al Consejo Nacional de Investigaciones Científicas y Técnicas, Buenos Aires.

BARRIENTOS, G., 1997. Nutrición y dieta de las poblaciones aborígenes prehispánicas del sudeste de la región pampeana. Tesis doctoral inédita. Facultad de Ciencias Naturales y Museo de La Plata. Universidad Nacional de La Plata, La Plata.

BARROS, P. y P. MESSINEO, 2004. Identificación y aprovisionamiento de chert o ftanita en la cuenca superior del arroyo Tapalqué (Olavarría, provincia de Buenos Aires, Argentina). Estudios Atacameños 28: 87-103.

BAYON, C. y N. FLEGENHEIMER, 1998. Un caso de aplicación: Procedencia de rocas en sitio El Guanaco. Resúmenes del Primer Congreso Nacional de Arqueología Pampeana, pp. 37, Venado Tuerto.
BAYON C. y C. ZAVALA, 1997. Coastal sites in south Buenos Aires: A review of Piedras Quebradas. En Quaternary of South America and Antarctic Peninsula, vol. 10 (1994), J. Rabassa y M. Salemme (Eds.), pp. 229-253. A. A. Balkema, Bookfield, Rotterdam.

BAYON, C., N. FLEGENHEIMER, M. VALENTE y A. PUPIO, 1999. Dime cómo eres y te diré de dónde vienes: La procedencia de rocas cuarcíticas en la región pampeana. Relaciones de la Sociedad Argentina de Antropología XXIV: 187-235.

BAYON, C., N. FLEGENHEIMER y M. I. GONZALEZ DE BONAVERI, 2000 Ms. Movimiento de rocas, movimiento de gente: Los mecanismos sociales de traslado de rocas. Ponencia presentada al Segundo Congreso Nacional de Arqueología Pampeana, pp. 37. Venado Tuerto.

BAYON, C., N. FLEGENHEIMER, M. ZARATE y C. DESCHAMPS, 2004. “...Y vendrán los arqueólog os en busca de un hueso"... sitio El Guanaco, partido de San Cayetano. En Aproximaciones arqueológicas pampeanas. 
Teorías, métodos y casos de aplicación contemporáneas, G. Martínez, M. Gutiérrez, R. Curtoni, M. Berón y P. Madrid (Eds.). Facultad de Ciencias Sociales, Universidad Nacional del Centro de la Provincia de Buenos Aires, Olavarría. En prensa.

BINFORD, L., 1979. Organization and formation processes: Looking at curated technologies. Journal of Anthropological Research 35 (3): 255-273.

BIRD, J., 1970. Paleoindian discoidal stones from southern South America. American Antiquity 35 (2): 205-209.

BONOMO, M., 2002. Distribución espacial y tecnología en el litoral marítimo bonaerense. En Del mar a los salitrales. Diez mil años de historia pampeana en el umbral del tercer milenio, D. Mazzanti, M. Berón y F. Oliva (Eds.), pp. 185-204. Universidad Nacional de Mar del Plata, Mar del Plata.

BORRERO L. y N. FRANCO, 1997. Early Patagonian huntergatherers: Subsistence and technology. Journal of Anthropological Research 53: 219-239.

FLEGENHEIMER, N., 1991. La Liebre, un sitio cantera-taller. Boletín del Centro 2: 58-64.

2001. Biface transport in the pampean region. Current Research in the Pleistocene 8: 21-22.

FLEGENHEIMER, N. y C. BAYON, 1999. Abastecimiento de rocas en sitios pampeanos tempranos: Recolectando colores. En En los tres reinos: Prácticas de recolección en el cono sur de América, C. Aschero, A. Korstanje, P. Vuoto (Eds.), pp. 95-107. Ediciones Magna Publicaciones, Tucumán.

2002. Cómo, cuándo y dónde. Estrategias de abastecimiento lítico en la pampa bonaerense. En Del mar a los salitrales. Diez mil años de historia pampeana en el umbral del tercer milenio, D. Mazzanti, M. Berón y F. Oliva (Eds.), pp. 231-241. Universidad Nacional de Mar del Plata, Mar del Plata.

FLEGENHEIMER, N. y M. ZARATE, 1997. Considerations on radiocarbon and calibrated dates from Cerro La China and Cerro El Sombrero, Argentina. Current Research in the Pleistocene 14: 27-28.

FLEGENHEIMER, N., C. BAYON y M. I. GONZALEZ DE BONAVERI, 1995. Técnica simple, comportamientos complejos: La talla bipolar en la arqueología bonaerense. Relaciones de la Sociedad Argentina de Antropología $\mathrm{XX}: 81-110$.

FLEGENHEIMER, N., S. KAIN, M. ZARATE y A. BARNA, 1996. Aprovisionamiento de cuarcitas en Tandilia, las canteras del Arroyo Diamante. Arqueología 6: 117-141.

FLEGENHEIMER, N., M. ZARATE y M. VALENTE, 1999. El área de canteras Arroyo Diamante, Barrer, Sierras de Tandil. Actas del XII Congreso Nacional de Arqueología Argentina, pp. 134-138. La Plata.
FLEGENHEIMER, N., R. GUICHON y C. SCABUZZO, 2002. Restos óseos humanos en el sitio El Guanaco, partido de San Cayetano. En Del mar a los salitrales. Diez mil años de historia pampeana en el umbral del tercer milenio, D. Mazzanti, M. Berón y F. Oliva (Eds.), pp. 121-126. Universidad Nacional de Mar del Plata, Mar del Plata.

FLEGENHEIMER, N., C. BAYON, M. VALENTE, J. BAEZA y J. FEMENINAS, 2003. Long distance tool stone transport in the Argentine Pampas. Quaternary International, The Journal of the INQUA, L. Miotti y M. Salemme (Eds.), vol. 109-110: 49-64.

FLEGENHEIMER, N., D. AMICK y C. BAYON, 2004. Early strategies of raw material acquisition and use in the southern cone. Journal of Anthropological Research. En prensa.

FRANCO, N., 1994, Maximización en el aprovechamiento de los recursos líticos. Un caso analizado en el área interserrana bonaerense. Arqueología Contemporánea 5: $75-88$.

GONZALEZ DE BONAVERI, M. I., 2002. Los cazadores recolectores pescadores de la cuenca inferior del río $\mathrm{Sa}$ lado (región pampeana). Tesis doctoral inédita, Facultad de Filosofía y Letras, Universidad de Buenos Aires, Buenos Aires.

GONZALEZ DE BONAVERI, M. I., M. FRERE, C. BAYON y N. FLEGENHEIMER, 1999. La organización de la tecnología en la cuenca del Salado. Arqueología 8: 57-76.

JESKE, R., 1992. Energetic efficiency and lithic technology: An Upper Mississippian example. American Antiquity 57 (3): 467-481.

KOLDEHOFF, B., 1987. The Cahokia flake tool industry: Socioeconomic implications for Late Prehistory in the Central Mississippi Valley. En The organization of core technology, J. K. Johnson y C. A. Morrow (Eds.), pp. 151-185. Westview Press, Boulder.

KUHN, S., 1995. Mousterian lithic technology. An ecological perspective. Princeton University Press, Princeton.

LOZANO, P., 1991. Cerro Aguirre: Un sitio de aprovisionamiento de materia prima lítica en la localidad de Sierras Bayas (provincia de Buenos Aires). Shincal 3: 145-149.

MADRID, P. y M. SALEMME, 1991. La ocupación tardía del sitio 1 de la Laguna Tres Reyes, Adolfo González Chaves, provincia de Buenos Aires. Boletín del Centro 3: $165-179$.

MADRID, P., G. POLITIS y D. POIRE, 2000. Pinturas rupestres y estructuras de piedra en las Sierras de Curicó (extremo noroccidental de Tandilia, región pampeana). Intersecciones en Antropología 1 (1): 35-53.

MARTINEZ, G., 1999. Tecnología, subsistencia y asentamientos en el curso medio del río Quequén Grande: Un enfoque arqueológico. Tesis doctoral inédita, Fa- 
cultad de Ciencias Naturales y Museo de La Plata, Universidad Nacional de La Plata, La Plata.

MARTINEZ, G. y Q. MACKIE, 2003/2004. Late Holocene human occupation of the Quequén Grande River Valley bottom: Settlement systems and an example of a built environment in the Argentine Pampas. Before Farming 1: $1-27$

MAZZANTI, D., 1993. Investigaciones arqueológicas en el sitio Cueva Tixi (provincia de Buenos Aires). Etnia 38-39: 125-163.

MAZZIA, N., C. SCABUZZO y R. GUICHON, 2004. Sobre cráneos, "caderas" y otros huesos. Entierros humanos en el sitio El Guanaco. En Aproximaciones arqueológicas pampeanas. Teorías, métodos y casos de aplicación contemporáneas, G. Martínez, M. Gutiérrez, R. Curtoni, M. Berón y P. Madrid (Eds.). Facultad de Ciencias Sociales, Universidad Nacional del Centro de la Provincia de Buenos Aires, Olavarría. En prensa.

MELTZER, D., 1989. "Was stone exchanged among eastern North American Paleoindians?". En Eastern Paleoindian lithic resource use, C. Ellis y J. Lothrop (Eds.), pp. 11-39. Westview Press, Boulder.

MESSINEO, P., 2002. Primeros resultados arqueológicos en la cuenca del arroyo Tapalqué (partido de Olavarría, provincia de Buenos Aires). En Del mar a los salitrales. Diez mil años de historia pampeana en el umbral del tercer milenio, D. Mazzanti, M. Berón y F. Oliva (Eds.), pp. 301-309. Universidad Nacional de Mar del Plata, Mar del Plata.

MESSINEO, P., P. BARROS, D. POIRE, y L. GOMEZ PERAL, 2002. Características litológicas de los niveles de chert o ftanitas en las Sierras Bayas (partido de Olavarría, provincia de Buenos Aires. Resúmenes CARPA 3: 89.

MIOTTI, L. y R. CATTANEO, 2003. Variations in strategies of lithic production and faunal exploitation in the Pleistocene/Holocene transition at Piedra Museo and surrounding region, L. Miotti, M. Salemme y N. Flegenheimer (Eds.), pp.105-111. Center for the Study of the First Americans-Texas A\&M University Press, College Station.

MOIRANO, J., 2000. Aprovisionamiento de recursos líticos y variabilidad artefactual en el sur de la subregión Pampa Húmeda: La revisión de las colecciones particulares. Relaciones de la Sociedad Argentina de Antropología XXIV: 237-255.
NAMI, H., 1999. Comentario. Relaciones de la Sociedad Argentina de Antropología XXIV: 223-229.

ODELL, G., 1996. Economizing behavior and the concept of "curation". En Stone tools. Theoretical insights into human prehistory, G. Odell (Ed.), pp. 51-80. Plenum Press, Nueva York.

OLIVA F. y G. BARRIENTOS, 1988. Laguna de Puan: Un potencial sitio de aprovisionamiento de materia prima lítica. Resúmenes del IX Congreso Nacional de Arqueología Argentina, p. 47. Buenos Aires.

OLIVA F. y J. MOIRANO, 1997. Primer informe sobre aprovisionamiento primario de riolita en Sierra de la Ventana (Pcia. de Buenos Aires, Argentina). En Arqueología pampeana en la década de los '90, M. Berón y G. Politis (Eds.), pp. 137-146. Museo de Historia Natural de San Rafael - Investigaciones arqueológicas y paleontológicas del cuaternario pampeano (INCUAPA) - Universidad Nacional del Centro de la Provincia de Buenos Aires, Mendoza.

ORMAZABAL, P., 1999. Lumb: Un sitio de aprovisionamiento de materia prima lítica para elementos de molienda. Actas XII Congreso Nacional de Arqueología Argentina, T. III: 156-164. La Plata.

PARRY, W. J. y R. L. KELLY, 1987. Expedient core technology and sedentism. En The organization of core technology, J. K. Johnson y C. A. Morrow (Eds.), pp. 285-304. Westview Press, Boulder.

POLITIS, G., 1984. Arqueología del área interserrana bonaerense. Tesis doctoral inédita. Facultad de Ciencias Naturales y Museo, Universidad Nacional de La Plata. La Plata.

POLITIS, G. y P. MADRID, 2001. Arqueología pampeana: Estado actual y perspectivas. En Historia argentina prehispánica, E. Berberián y A. Nielsen (Eds.), T. II: 737813. Editorial Brujas, Córdoba.

PUPIO, A., 1996. Resultados preliminares del sitio canterataller La Liebre. Jornadas Chivilcoyanas en Ciencias Sociales y Naturales, pp. 191-194. Chivilcoy.

VALVERDE, F, 2002. Variabilidad de recursos líticos en dos sitios paleoindios de las sierras de Tandilia oriental, provincia de Buenos Aires. En Del mar a los salitrales. Diez mil años de historia pampeana en el umbral del tercer milenio, D. Mazzanti, M. Berón y F. Oliva (Eds.), pp. 281287. Universidad Nacional de Mar del Plata, Mar del Plata. 